\title{
The Utility of Repeat Midtrimester Anatomy Ultrasound for Anomaly Detection
}

\author{
S. Lindsay Wood, $\mathrm{MD}^{1} \quad$ John Owen, MD, $\mathrm{MSPH}^{1} \quad$ Sheri M. Jenkins, $\mathrm{MD}^{1} \quad$ Lorie M. Harper, MD, $\mathrm{MSCl}^{1}$ \\ ${ }^{1}$ Center for Women's Reproductive Health, Department of Obstetrics \\ and Gynecology, University of Alabama at Birmingham, \\ Birmingham, Alabama \\ Address for correspondence S. Lindsay Wood, MD, Division of Maternal- \\ Fetal Medicine, Department of Obstetrics and Gynecology, University of \\ Alabama at Birmingham, 619, 19th Street S \# 176F, STE 10270, \\ Birmingham, AL 35249-1900 (e-mail: Iwood@hrpregnancy.com).
}

Am J Perinatol 2018;35:1346-1351.

\begin{abstract}
Introduction Although guidelines recommend repeat ultrasound in the setting of an incomplete fetal anatomic survey, the clinical utility of this practice has not been established. As such, we aimed to assess the yield of repeat ultrasound for anomaly detection following an incomplete survey.

Materials and Methods This is a retrospective cohort study of all singletons who underwent a midtrimester anatomic ultrasound at University of Alabama at Birmingham (UAB) from 2006 to 2014. Patients with an incomplete ultrasound underwent repeat examinations until completion. The population was divided into cohorts FIRST, SECOND, and THIRD, corresponding to the ultrasound at which the exam was deemed

\section{Keywords}

- incomplete ultrasound

- suboptimal ultrasound visualization

- repeat anatomy ultrasound

- anatomy scan

- anomaly detection complete. Each detected anomaly was tallied. The number of ultrasounds needed to detect an anomaly was then assessed per group.

Results Of 15,768 ultrasounds performed on 13,740 patients, 11,828 exams were completed on first attempt; 1,796 patients required a second, while 116 patients required a third scan or more. We detected 324 anomalies; $93.8 \%$ in FIRST, 5.9\% in SECOND, and $0.3 \%$ in THIRD. The number of scans needed to detect an anomaly was 39 , 189, and 348 for FIRST, SECOND, and THIRD, respectively.

Conclusion Over $90 \%$ of anomalies are detected on the initial fetal anatomic survey. The incremental diagnostic yield then decreases, requiring appreciably more repeat scans to detect one anomaly.
\end{abstract}

According to the Eunice Kennedy Shriver National Institute of Child Health and Human Development (NICHD) Joint Workshop Executive Summary on fetal imaging, the ideal timing for a single obstetric ultrasound is 18 to 20 weeks' gestation, allowing for optimal evaluation of fetal anatomy with sufficient clarity to detect many major malformations. ${ }^{1}$ The International Society of Ultrasound in Obstetrics and Gynecology (ISUOG) Clinical Standards Committee also advocates for performance of the midtrimester scan between 18 and 22 weeks, ensuring timely detection of major structural anomalies. $^{2}$ As such, the midtrimester anatomy scan has become a routine component of prenatal care in developed

received

April 15, 2017

accepted after revision

December 29, 2017

published online

February 8, 2018 countries, particularly given that most anomalous fetuses are born to women with no identifiable risk factors. ${ }^{1,3}$ Although the sensitivity of the midtrimester anatomy ultrasound has been debated, some tertiary centers have reported a detection rate of over $80 \%$ for major anomalies. ${ }^{3}$

To complete a standard or detailed ultrasound examination of fetal anatomy, multiple key structures, as defined by accepted practice guidelines, must be visualized ( - Figs. $\mathbf{1}$ and 2). The reported sensitivity applies to an ultrasound in which all essential components are visualized. Despite this, completion of the anatomic survey may not always be feasible on the first exam, with completion rates as low as
Copyright $\odot 2018$ by Thieme Medical Publishers, Inc., 333 Seventh Avenue, New York, NY 10001, USA. Tel: +1(212) 584-4662.
DOI https://doi.org/ 10.1055/s-0038-1626715. ISSN 0735-1631. 


\begin{tabular}{|c|c|c|}
\hline Bead/face/Nek & Chest & Spine \\
\hline \multirow{5}{*}{$\begin{array}{c}\text { Lateral cerebral ventricles } \\
\text { Choroid plexus } \\
\text { Midline falx } \\
\text { Cavum septi pellucidi } \\
\text { Cerebellum } \\
\text { Cisterna magna } \\
\text { Upper lip } \\
\text { *measurement of the nuchal fold } \\
\text { may be helpful during a specific age } \\
\text { interval to suggest an increased risk } \\
\text { of aneuploidy }\end{array}$} & $\begin{array}{c}\text { Heart (with cardiac activity) } \\
\text { 4-chamber view } \\
\text { Left ventricular outflow } \\
\text { tract } \\
\text { Right ventricular outflow } \\
\text { tract }\end{array}$ & $\begin{array}{c}\text { Cervical } \\
\text { Thoracic } \\
\text { Lumbar } \\
\text { Sacral }\end{array}$ \\
\hline & Abdoment & Extremities \\
\hline & $\begin{array}{c}\text { Stomach (presence, size, \& } \\
\text { situs) } \\
\text { Kidneys } \\
\text { Urinary bladder } \\
\text { Umbilical cord insertion site }\end{array}$ & $\begin{array}{l}\text { Legs } \\
\text { Arms }\end{array}$ \\
\hline & into fetal abdomen & Sex \\
\hline & $\begin{array}{l}\text { Umbilical cord vessel } \\
\text { number }\end{array}$ & $\begin{array}{l}\text { In multiple gestations and when } \\
\text { medically indicated }\end{array}$ \\
\hline
\end{tabular}

Fig. 1 Elements of the standard fetal anatomic examination. Shown are the minimal elements of a standard anatomic ultrasound as endorsed by the American College of Radiology (ACR), the American College of Obstetricians and Gynecologists (ACOG), American Institute of Ultrasound in Medicine (AIUM), and the Society of Radiologists in Ultrasound (SRU). ${ }^{3,13,14}$

$31 \%$ reported in the literature. ${ }^{4}$ Several factors may account for suboptimal ultrasound visualization, including early gestational age, fetal position, oligohydramnios, prior abdominal surgery, and maternal obesity. ${ }^{4-7}$

Although accepted guidelines recommend follow-up with repeat ultrasound in the setting of an incomplete anatomic survey, the clinical utility and cost-effectiveness of this practice have not yet been established. ${ }^{1,2}$ Undoubtedly, subjecting patients to repeat ultrasound examinations presents challenges of additional time and expense and may be seen as burdensome both to the health care system and the patient. Further, the incremental diagnostic utility of repeat ultrasound for anomaly detection is unknown, limiting the counseling on its necessity. As such, we aimed to assess the yield of repeat anatomic ultrasound for anomaly detection following a prior incomplete anatomic evaluation. We hypothesized that the diagnostic yield, or number of anomalous fetuses detected per scan, would decrease with each scan performed.

\section{Materials and Methods}

We conducted a retrospective cohort study of all singletons who underwent a fetal anatomic survey between 16 and 20 weeks' gestation at a single tertiary center, the University of Alabama at Birmingham, from 2006 to 2014. Institutional review board approval with waiver of consent was obtained. Fetal deaths occurring prior to 20 weeks' gestation were excluded from analysis. It is routine practice at our institution to repeat the anatomic survey every 3 to 4 weeks until

\begin{tabular}{|c|c|c|}
\hline Intracromialyfacial/Spinal & Chast & Limbs \\
\hline \multirow{7}{*}{$\begin{array}{c}\text { Lateral ventricles*, } 3^{\text {rd }} \text { \& } 4^{\text {th }} \\
\text { ventricles } \\
\text { Cerebellum*, integrity of lobes*, } \\
\text { vermis* } \\
\text { Cavum septum pellucidum } \\
\text { Cisterna magna measurement* } \\
\text { Nuchal thickness measurement } \\
\text { (15-20 weeks)* } \\
\text { Integrity of cranial vault } \\
\text { Examination of brain } \\
\text { parenchyma (e g. for } \\
\text { calcifications) } \\
\text { Ear position, size } \\
\text { Face } \\
\text { Upper lip integrity* } \\
\text { Palate* } \\
\text { Facial profile* } \\
\text { of the neck (e g. for } \\
\text { masses) }\end{array}$} & $\begin{array}{c}\text { Presence of masses* } \\
\text { Pleural effusion* } \\
\text { Integrity of both sides of the } \\
\text { diaphragm* } \\
\text { Appearance of ribs }\end{array}$ & $\begin{array}{l}\text { Number, size, \& architecture* } \\
\text { Anatomy and position of hands* } \\
\text { Anatomy and position of feet* }\end{array}$ \\
\hline & Feart & Placenta and cord \\
\hline & $\begin{array}{c}\text { Cardiac location \& axis* } \\
\text { Outflow tracts* }\end{array}$ & $\begin{array}{l}\text { Placental cord insertion site* } \\
\text { Placental masses* } \\
\text { Umbilical cord (number of } \\
\text { vessels) }\end{array}$ \\
\hline & Abdoment & Anmiotic fluid \\
\hline & $\begin{array}{c}\text { Bowel* } \\
\text { Adrenal glands } \\
\text { Gallbladder } \\
\text { Liver } \\
\text { Spleen } \\
\text { Ascites* } \\
\text { Masses }\end{array}$ & $\begin{array}{l}\text { Assessment of Amuiotic Fluid } \\
\text { Volume* }\end{array}$ \\
\hline & Gemitatia & Maternal anatomy \\
\hline & Gender & $\begin{array}{c}\text { Evaluation of cervix (not } \\
\text { required) } \\
\text { Evaluation of maternal adnexa } \\
\text { when feasible* }\end{array}$ \\
\hline
\end{tabular}

Fig. 2 Elements of the detailed fetal anatomic examination. Shown are the components to a detailed anatomic ultrasound as endorsed by the Society for Maternal-Fetal Medicine (SMFM). ${ }^{15}$ Of note, the detailed examination also includes all elements of the standard examination. Components considered integral to the exam are denoted by *. 
completed. Of note, we only included patients whose indication for second referral was incomplete anatomy. As the goal of this study was not to evaluate the utility of fetal echocardiography, these scans were not included. Regarding inclusion of anatomy scans prior to 18 weeks, patients are frequently referred to our center and scheduled as early as 16 weeks, either due to no prior dating scan or scheduling limitations. As 16 weeks' gestation is the earliest we would attempt an anatomy scan, we included these patients in our analysis.

The study population was identified through our department's current ultrasound picture archive and communication system, AS-OBGYN (AS Software Inc., Englewood Cliffs, $\mathrm{NJ}$ ), which was implemented in 2005. As documentation during the implementation phase of AS-OBGYN may have led to initial inconsistencies, we chose to begin the study period in 2006 to reduce information bias. In the AS-OBGYN system, elements of the ultrasound are documented in the report as "normal," "visualized," "abnormal," "visualized on color Doppler," "not visualized," “poorly visualized," or "normal/ abnormal on limited views," and the system can be queried using these terms.

Completion of the anatomy ultrasound, either standard or detailed, was defined by criteria from the American Institute of Ultrasound in Medicine (AIUM), the Society for MaternalFetal Medicine (SMFM), the American College of Radiology (ACR), the Society of Radiologists in Ultrasound (SRU), and the American College of Obstetricians and Gynecologists (ACOG) (-Figs. 1 and 2). All standard ultrasounds were performed by registered diagnostic medical sonographers and interpreted by Maternal-Fetal Medicine (MFM) faculty in AIUM-accredited facilities; detailed ultrasounds were personally performed or supervised by the MFM faculty. Sonographic images were captured using General Electric Voluson E8, 730 Expert or 730 Pro units (GE Healthcare, Waukesha, WI) and stored in AS-OBGYN.

An ultrasound was considered incomplete if any of the required anatomical elements were documented as "not visualized," "poorly visualized," or "normal/abnormal on limited views." Conversely, an ultrasound was considered complete if structures were "normal," "visualized," "abnormal," or "visualized on color Doppler." Although we do not routinely incorporate color Doppler into all fetal anatomic surveys, if this modality was used to successfully image a structure not well seen on two-dimensional (2D), that anatomic element was deemed complete. Patients with an incomplete examination underwent repeat exams until the anatomic survey was completed. For the purpose of this study, if an anomaly was detected, the exam was deemed complete on that ultrasound, as the endpoint of anomaly detection was met. Anomaly was defined as any structural malformation that altered care during the pre- or postnatal period (e.g., prompting follow-up ultrasound and/or possible invasive testing). Of note, isolated soft markers for aneuploidy (e.g., echogenic intracardiac foci and choroid plexus cysts) were excluded with the exceptions of thickened nuchal fold and echogenic bowel, as these findings prompt follow-up and/or invasive prenatal diagnosis in our practice.
The study population was divided into three cohorts: patients whose ultrasound exam was completed on the first attempt (FIRST), those whose exam was completed on the second ultrasound (SECOND), and those who required a third ultrasound or more (THIRD). Outcomes of interest were as follows: (1) the number of anomalous fetuses detected in each of the three cohorts, (2) the number of ultrasounds needed to detect one anomaly (defined as [number of patients undergoing ultrasound $\times$ number of ultrasound attempts]/anomalous fetuses detected per cohort), and ( 3 ) the rate of congruence between anomalies detected on repeat ultrasound and previously suboptimally visualized system on prior ultrasound. We compared selected patient characteristics and comorbidities among the three cohorts using Pearson's chi-square and analysis of variance (ANOVA), where appropriate, with a two-tailed $p$ $\leq 0.05$ considered significant. Data were analyzed utilizing StataSE 13 (StataCorp LP, College Station, TX).

\section{Results}

We identified 13,740 subjects who underwent a total of 15,768 anatomic ultrasounds during the study period. Of these, 2,315 patients underwent a detailed anatomy ultrasound, whereas 11,425 underwent a standard anatomy ultrasound. Of the 13,740 subjects, 11,828 (86\%) patients' ultrasounds were completed on first attempt at a mean gestational age of 18.5 ( \pm 0.9 ) weeks (FIRST). A second scan was required in 1,796 (13\%) patients at a mean of 19.7 ( \pm 2.4 ) weeks (SECOND). One hundred and sixteen (1\%) patients required a third ultrasound or more for anatomy completion at a mean of $20.4( \pm 2.9)$ weeks (THIRD; - Fig. 3).

As the number of ultrasounds increased, patients were significantly more likely to be older, obese, have chronic hypertension and/or pregestational diabetes, and to have an earlier gestational age at the initial ultrasound attempt $(p<0.001 ;-$ Table 1). The three groups also differed by race and payer, with an increased proportion of white, privately insured patients undergoing repeat ultrasound $(p<0.001 ;$ - Table 1).

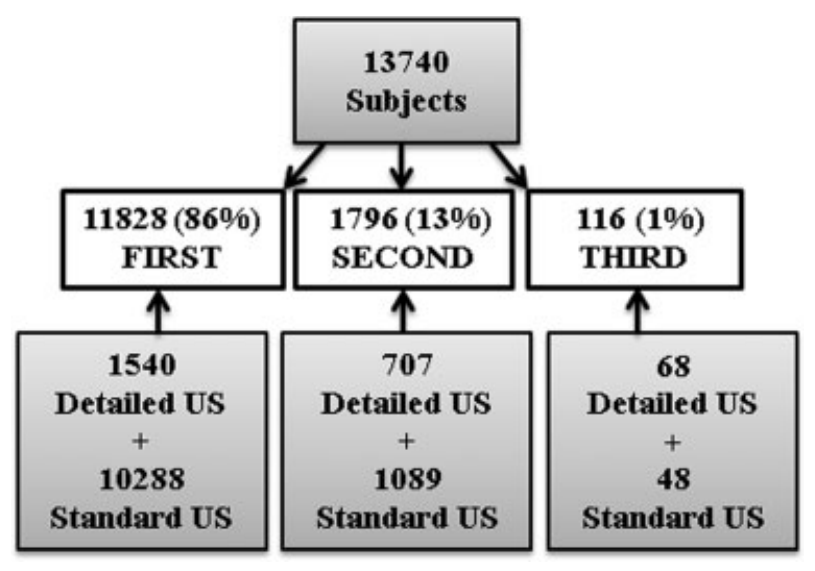

Fig. 3 Study population. This flowchart illustrates the total patient population along with the groups as defined in the text. Noted below the groups are numbers of patients who underwent detailed versus standard ultrasounds (US) within each group. 
Table 1 Demographics and selected comorbidities of the study population, stratified by the number of ultrasound examinations until completion

\begin{tabular}{|l|l|l|l|l|}
\hline & FIRST $(\boldsymbol{n}=\mathbf{1 1 , 8 2 8})$ & SECOND $(\boldsymbol{n}=\mathbf{1 , 7 9 6})$ & THIRD $(\boldsymbol{n}=\mathbf{1 1 6})$ & $p$-Value \\
\hline Age $(\mathrm{y})$ & $24.7( \pm 5.5)$ & $27.0( \pm 6.6)$ & $27.5( \pm 6.4)$ & $<0.001$ \\
\hline Gestational age at first ultrasound $(\mathrm{wk})$ & $18.5( \pm 0.9)$ & $17.8( \pm 1.3)$ & $17.6( \pm 1.3)$ & $<0.001$ \\
\hline Race/Ethnicity & & & & $<0.001$ \\
\hline White & $1,923(16.3)$ & $394(21.9)$ & $34(29.3)$ \\
\hline Black & $7,399(62.6)$ & $1,019(56.7)$ & $71(61.2)$ \\
\hline Hispanic & $2,296(19.4)$ & $339(18.9)$ & $11(9.5)$ \\
\hline Asian & $49(0.4)$ & $10(0.6)$ & $0(0)$ \\
\hline Other & $161(1.3)$ & $34(1.9)$ & $0(0)$ \\
\hline Payer & & & \\
\hline Government & $7,767(65.7)$ & $1,146(63.8)$ & $70(60.3)$ \\
\hline Private & $833(7.0)$ & $217(12.1)$ & $21(18.1)$ \\
\hline Self & $3,137(26.5)$ & $403(22.4)$ & $21(18.1)$ \\
\hline Unknown & $91(0.8)$ & $30(1.7)$ & $4(3.5)$ & $<0.001$ \\
\hline BMI (kg/m $\left.{ }^{2}\right)$ & $28.3( \pm 7.9)$ & $30.9( \pm 9.6)$ & $34.3( \pm 10.9)$ & $<0.001$ \\
\hline Pregestational diabetes mellitus & $342(2.9)$ & $195(10.9)$ & $23(19.8)$ \\
\hline Chronic hypertension & $1,890(16.0)$ & $447(24.9)$ & $35(30.2)$ & $<0.001$ \\
\hline
\end{tabular}

Abbreviations: ANOVA, analysis of variance; BMI, body mass index.

Note: Data are presented as a mean ( \pm standard deviation) or $n(\%)$. Statistical tests used: ANOVA or Pearson's chi-square test, as appropriate.

In total, 324 anomalies were detected in the cohort, equating to a $2.4 \%$ prevalence of congenital anomalies, which is consistent with the reported baseline rate. ${ }^{8}$ The majority of anomalies (93.8\%) were detected in FIRST, 5.9\% in SECOND, and $0.3 \%$ in THIRD. A total of 20 anomalies were detected on repeat ultrasound exams; in SECOND, several severe anomalies were diagnosed, including hypoplastic left heart syndrome (HLHS), hydrocephalus, and encephalocele. However, the only anomaly detected in THIRD was a two-vessel umbilical cord (-Table 2 ).

We performed 11,828 ultrasounds to detect the 304 anomalies identified on the first ultrasound; therefore, the

Table 2 Anomalies detected on repeat ultrasound

\begin{tabular}{|l|}
\hline Two-vessel umbilical cord (6) \\
\hline Ventriculomegaly (3) \\
\hline Hydrocephalus (2) \\
\hline Hypoplastic left heart (2) \\
\hline Echogenic bowel (3) \\
\hline Abdominal umbilical cord insertion (1) \\
\hline Dandy-Walker variant (1) \\
\hline Hypospadias (1) \\
\hline Encephalocele (1) \\
\hline
\end{tabular}

Note: Shown are the type and number of anomalies detected on repeat ultrasound. In total, 20 anomalies were detected on repeat ultrasound, with only one, a two-vessel umbilical cord, detected in those undergoing a THIRD ultrasound or more. number needed to scan to detect one anomaly in FIRST was 39. A total of 1,796 women underwent two scans to detect an additional 19 anomalies, for a number needed to scan to detect one anomaly in SECOND of 189 . We detected only one anomaly in THIRD; considering 116 women underwent 3 ultrasounds to detect this one anomaly, the number needed to scan to detect one anomaly in this group was 348 .

When an anomaly was detected on repeat ultrasound, the system in which the anomaly was located was mostly congruent with the previously suboptimally visualized system on the initial incomplete ultrasound ( $p=0.044 ;-$ Table 3$)$. If the

Table 3 When an anomaly is detected on repeat ultrasound, is it congruent or incongruent with the suboptimally visualized system on first ultrasound?

\begin{tabular}{|l|l|l|l|}
\hline Anomaly type & $\begin{array}{l}\text { Congruent } \\
\text { SUV } \\
\text { system }\end{array}$ & $\begin{array}{l}\text { Incongruent } \\
\text { SUV system }\end{array}$ & -Value \\
\hline Head/Face/Spine & 5 & 0 & \multirow{2}{*}{$0.044^{\mathrm{a}}$} \\
\cline { 1 - 3 } Chest/Heart & 3 & 1 & \\
\hline $\begin{array}{l}\text { Abdomen/ } \\
\text { Genitourinary/ } \\
\text { Gastrointestinal }\end{array}$ & 4 & 7 & \\
\cline { 1 - 3 } Extremities & 0 & 0 & \\
\cline { 1 - 2 } $\begin{array}{l}\text { All anomaly } \\
\text { types }\end{array}$ & 12 & 8 & \\
\hline
\end{tabular}

Abbreviation: SUV, suboptimally visualized.

${ }^{\mathrm{a} C h i}$-square test. 
patient was appointed for repeat ultrasound secondary to incomplete visualization of the fetal abdominal, genitourinary, or gastrointestinal systems, the anomaly subsequently detected was more likely to be in a different system ( - Table 3 ).

\section{Conclusion}

In our study, over $90 \%$ of anomalies were detected on the initial fetal anatomic survey. Following this, the incremental yield decreased, requiring appreciably more scans to detect one anomaly. We observed a number needed to scan to detect an anomaly of 39, 189, and 348 for those undergoing, respectively, one, two, or three or more scans for survey completion. These findings are consistent with our hypothesis that the diagnostic yield, or number of anomalous fetuses detected per scan, decreases with each scan performed. We further observed that when an anomaly is detected on repeat ultrasound, this is more likely to be detected in a system that was previously suboptimally visualized.

Applying the average cost of a detailed (\$1,100 USD) or standard (\$425 USD) ultrasound at University of Alabama at Birmingham (UAB) to our cohort, $\$ 2.8$ million USD were spent to detect the 20 anomalies identified in patients undergoing repeat ultrasound; of this, roughly $\$ 286,000$ USD was spent to detect the one anomaly, a two-vessel umbilical cord, in patients undergoing three or more ultrasounds. This estimate omits patient travel, anxiety, prenatal genetic screening, invasive diagnosis, and lost income, among other factors. In an era of cost-containment within an overburdened health care system, one must question the incremental value of each repeat ultrasound given its diagnostic yield.

In a recent case-control study, Waller et al assessed the incidence of fetal anomalies following an incomplete anatomic survey, matching 1,030 incomplete and 1,030 complete scans. ${ }^{9}$ They reported a completion rate of $40 \%$ on a subsequent scan following an initial incomplete ultrasound, with a mean of two scans for completion. Five percent of these patients had a fetal anomaly or soft marker for aneuploidy detected on a subsequent scan. The authors recommended repeating the anatomic survey until complete. ${ }^{9}$ Our findings, however, suggest that this policy may not be a reasonable utilization of resources. Rather, counseling patients on the low residual risk of an anomaly or limiting the number of repeat scans to one, as was recommended in the NICHD Executive Summary, may be a more viable alternative. ${ }^{1}$

The effect of obesity on ultrasound completion, as observed in our cohort, is well-documented. ${ }^{4,6,7}$ In a study by Thornburg et al, only $17 \%$ of class III obese patients had a complete standard exam on first attempt; the completion rate was even lower (14\%) for those undergoing detailed ultrasound. The completion rates within this group only rose to 49 and $31 \%$, respectively, after a mean of 1.9 and 2.2 scans. The authors estimated that for every 100 obese women, an extra 33 scans would be required. These findings were supported in two studies by Dashe et al, which noted a significant decrease in ultrasound completion as body mass index (BMI) increased and a corresponding decrease in anomaly detection with increasing BMI. ${ }^{6,7}$ Obesity has been reported as a significant independent risk factor for most fetal structural anomalies, ${ }^{10}$ presenting a potential dilemma; this population is more likely to have an anomalous fetus but less likely to have a complete ultrasound. Given increasing rates of obesity in the United States, the completion rate of the anatomic ultrasound is likely to decrease over time. Importantly, our results were obtained in a generally overweight to obese population and still suggest that repeating the midtrimester ultrasound until completion may not be the most judicious approach.

Hendler et al reported two studies on suboptimal ultrasound visualization of fetal cardiac anatomy. ${ }^{5,11}$ In their more recent study of 372 patients undergoing repeat ultrasound, one cardiac anomaly (atrioventricular $[\mathrm{AV}]$ canal defect) was detected on repeat scan at a mean of 21.4 weeks' gestation. ${ }^{5}$ Persistent suboptimal cardiac views were present in $20 \%$ of their population with class III obesity. ${ }^{5}$ In their prior study, they examined the rate of persistent suboptimal anatomy visualization in a population of over 11,000 and showed no reduction in suboptimal visualization of cardiac anatomy in obese patients undergoing repeat ultrasound after 18 to 20 weeks' gestation. ${ }^{11}$ The findings of both of these studies are consistent with our finding of limited diagnostic yield of anomaly detection with repeat ultrasound. Related to their findings, we observed that when a chest/heart anomaly is detected on repeat ultrasound, this system was likely to be previously suboptimally visualized. In ours and Hendler et al's study, ${ }^{5}$ significant cardiac defects (HLHS, AV canal defect) were detected on follow-up ultrasound. Given the significance of these anomalies, our findings should be applied with caution, perhaps utilizing a triage system of the need for repeat ultrasound based on the system that was initially suboptimally visualized. Based upon their most recent findings, Hendler et al suggested that the benefit of detecting cardiac anomalies likely outweighs the risk of repeat ultrasound. ${ }^{5}$ Although the benefit of prenatally diagnosing certain anomalies (e.g., ductal-dependent lesions) is evident, it is unclear to what extent repeat anatomy ultrasound should be performed to meet this endpoint.

Limitations to our study include its reliance on retrospective data, introducing potential information and misclassification bias. Given our use of data recorded in the electronic medical record, we are unable to reliably ascertain the reason for the incomplete survey (e.g., fetal position, body habitus), as this is typically noted in a varied free-text field, which is unable to be standardized in a large database. Additionally, as our ultrasound clinics serve a large referral and consultative population, collection of delivery, newborn, and long-term follow-up data on all patients included in our study was not feasible, limiting our ability to assess the true residual risk of an anomaly following an incomplete survey. Further, any study assessing midtrimester anomaly detection cannot account for anomalies that may develop over time (e.g., bowel obstruction). As previously noted, we did include patients undergoing their initial anatomy ultrasound as early as 16 weeks, which is outside of the reported optimal timing of $18+$ weeks as defined by NICHD and ISUOG. ${ }^{1,2}$ 
Given the large referral network we serve, patients are frequently sent early and scheduled in this time range, either due to no prior dating scan or scheduling limitations. Although not optimal, this is likely more representative of everyday practice. We believe our experience and results can further aid referring and consulting providers in more optimal timing and completion of the midtrimester scan. Experienced institutions and those serving high-risk populations have been previously reported to have higher ultrasound sensitivities for fetal anomaly detection than less experienced centers. ${ }^{12}$ As our data come from a high-risk center, our population, ultrasound completion rate, and observed anomaly rates may not be generalizable to other institutions. However, when applied in a similar context, we feel that our experience may provide useful information in counseling patients and in utilization of resources.

To our knowledge, the required elements for completion of the anatomic survey did not differ from those summarized in $\boldsymbol{- F i g s .} \mathbf{1}$ and $\mathbf{2}$ during the course of our study. Additionally, our department's ultrasound picture archive and communication system did not differ in the study period, leading to consistent documentation of the same required elements throughout the study course. On review of the proportions of anatomic survey completion year-by-year (data not presented), these rates do not appear to vary or trend toward higher/lower completion rates through the course of the study.

Strengths of our study include its large, diverse, population, encompassing over 13,500 patients evaluated at a large obstetric tertiary center. Further, our institution's use of a standardized imaging protocol based upon accepted practice guidelines improves the reproducibility and application of our findings at other centers., ${ }^{3,13-15}$ Finally, our aim of assessing the incremental diagnostic yield of repeat ultrasound was met; importantly, this objective has been identified as an area of needed research by the NICHD, ${ }^{1}$ and we believe our results will aid in the management of an incomplete anatomic survey, which is likely to increase over time.

Based upon our findings, when approaching an incomplete fetal anatomic survey, we suggest that patients be counseled that most anomalies will be detected on initial anatomy ultrasound. Should a repeat ultrasound be performed secondary to suboptimal visualization, we recommend carefully surveying the previously suboptimally visualized systems, as detectable anomalies are more likely to be present in these. However, given the limited yield for anomaly detection and considering health care resources, we suggest that the use of repeat midtrimester ultrasound for anomaly detection-particularly the use of more than one repeat ultrasound-should be minimized.

\section{Note}

This study was presented in part as an oral presentation at the Annual Convention of the American Institute of Ultrasound in Medicine (AIUM), New York City, NY, USA, March 17-21, 2016.
Funding

None.

Conflict of Interest

None.

\section{References}

1 Reddy UM, Abuhamad AZ, Levine D, Saade GR; Fetal Imaging Workshop Invited Participants. Fetal imaging: executive summary of a Joint Eunice Kennedy Shriver National Institute of Child Health and Human Development, Society for Maternal-Fetal Medicine, American Institute of Ultrasound in Medicine, American College of Obstetricians and Gynecologists, American College of Radiology, Society for Pediatric Radiology, and Society of Radiologists in Ultrasound Fetal Imaging Workshop. Am J Obstet Gynecol 2014;210(05):387-397

2 Salomon LJ, Alfirevic Z, Berghella V, et al; ISUOG Clinical Standards Committee. Practice guidelines for performance of the routine mid-trimester fetal ultrasound scan. Ultrasound Obstet Gynecol 2011;37(01):116-126

3 American College of Obstetricians and Gynecologists. ACOG Practice Bulletin No. 175: ultrasonography in pregnancy. Obstet Gynecol 2016;128(06):241-256

4 Thornburg LL, Miles K, Ho M, Pressman EK. Fetal anatomic evaluation in the overweight and obese gravida. Ultrasound Obstet Gynecol 2009;33(06):670-675

5 Hendler I, Blackwell SC, Bujold E, et al. Suboptimal secondtrimester ultrasonographic visualization of the fetal heart in obese women: should we repeat the examination? J Ultrasound Med 2005;24(09):1205-1209

6 Dashe JS, McIntire DD, Twickler DM. Maternal obesity limits the ultrasound evaluation of fetal anatomy. J Ultrasound Med 2009; 28(08):1025-1030

7 Dashe JS, McIntire DD, Twickler DM. Effect of maternal obesity on the ultrasound detection of anomalous fetuses. Obstet Gynecol 2009;113(05):1001-1007

8 Whitworth M, Bricker L, Mullan C. Ultrasound for fetal assessment in early pregnancy. Cochrane Database Syst Rev 2015;14 (07):CD007058

9 Waller SA, O'Connell K, Carter A, et al. Incidence of fetal anomalies after incomplete anatomic surveys between 16 and 22 weeks. Ultrasound Q 2013;29(04):307-312

10 Stothard KJ, Tennant PW, Bell R, Rankin J. Maternal overweight and obesity and the risk of congenital anomalies: a systematic review and meta-analysis. JAMA 2009;301(06):636-650

11 Hendler I, Blackwell SC, Bujold E, et al. The impact of maternal obesity on midtrimester sonographic visualization of fetal cardiac and craniospinal structures. Int J Obes Relat Metab Disord 2004; 28(12):1607-1611

12 Levi S. Ultrasound in prenatal diagnosis: polemics around routine ultrasound screening for second trimester fetal malformations. Prenat Diagn 2002;22(04):285-295

13 American College of Radiology. ACR-ACOG-AIUM-SRU practice parameter for the performance of obstetrical ultrasound. (Amended 2014). Available at: http://www.acr.org/ /media/ f7bc35bd 59264e7cbe648f6d1bb8b8e2.pdf. Accessed February 2015

14 American Institute of Ultrasound in Medicine. AIUM practice guideline for the performance of obstetric ultrasound examinations. J Ultrasound Med 2013;32(06):1083-1101

15 Society for Maternal Fetal Medicine. Coding Committee. (Revised December 27, 2012). White Paper on Ultrasound Code 76811. Available at: https://www.smfm.org/attachedfiles/UltrasoundCode76811Revised-Dec272012.pdf. Accessed February 2015 